\title{
ENCLOSURE OF SOLUTIONS FOR ELLIPTIC BOUNDARY VALUE PROBLEMS WITH NONMONOTONE DISCONTINUOUS NONLINEARITY
}

\author{
SIEGFRIED CARL
}

\author{
Fachbereich Mathematik/Informatik, Martin-Luther-Universität
} Postfach 8, D-O-4010 Halle, Germany

Introduction. Let $\Omega \subset \mathbb{R}^{n}$ be a bounded domain with Lipschitz boundary $\partial \Omega$. In this paper we consider the elliptic boundary value problem (BVP)

$$
A u=f(u) \text { in } \Omega, \quad u=0 \quad \text { on } \partial \Omega,
$$

where $A$ is a uniformly elliptic differential operator of the form

$$
A u=-\frac{\partial}{\partial x_{i}}\left(a_{i j} \frac{\partial u}{\partial x_{j}}\right)
$$

with coefficients $a_{i j} \in L^{\infty}(\Omega)$. The nonlinearity $f: \mathbb{R} \rightarrow \mathbb{R}$ on the right-hand side of (1.1) may be discontinuous.

Semilinear elliptic equations of the form (1.1) involving discontinuous nonlinearities occur in various models of application, e.g. in the study of a single, irreversible steady state reaction of zero order where the reaction term $f$ is given by $f(s)=0$ if $s \leq 0$, and $f(s)=\lambda$ if $s>0$ for some positive constant $\lambda$ (cf. [1, $9]$ ). Another example is given by the model of relay (or on-off) control systems which involves the signum nonlinearity defined by $f(s)=s /|s|$ if $s \neq 0$, and $f(0)=0$ (cf. $[7,11])$. In recent years discontinuous nonlinearities have arisen also in a variety of free boundary problems.

It is well known that for continuous right-hand sides $f$ the existence of an upper solution $\bar{u}$ and a lower solution $\underline{u}$ of the BVP (1.1) satisfying $\bar{u} \geq \underline{u}$ implies the existence of solutions between them (cf. [8]). If the right-hand side $f$ is only one-sided continuous but is supposed to satisfy the condition

$$
f\left(s_{1}\right)-f\left(s_{2}\right) \geq-M\left(s_{1}-s_{2}\right) \quad \text { for some } M \geq 0 \text { and } s_{1} \geq s_{2},
$$

1991 Mathematics Subject Classification: 35J20, 35R05, 49A29.

The paper is in final form and no version of it will be published elsewhere. 
then the existence of the greatest (resp. smallest) solution of the BVP (1.1) within the interval $[\underline{u}, \bar{u}]$ can be proved by monotone iteration (cf. [4]). Finally, the assumption of one-sided continuity can completely be removed by applying a generalized iteration method due to Heikkilä (cf. $[5,13]$ ), which, however, can hardly be used for computational purposes.

The aim of this paper is to prove the existence of solutions of the BVP (1.1) lying between upper and lower solutions in the case that the discontinuous nonlinear right-hand side $f$ satisfies the following hypothesis:

(H1) There exists a monotone nondecreasing function $h: \mathbb{R} \rightarrow \mathbb{R}$ such that the function $g: \mathbb{R} \rightarrow \mathbb{R}$ defined by

$$
g(s):=f(s)+h(s)
$$

is nondecreasing and either right continuous for all $s \in \mathbb{R}$ or left continuous for all $s \in \mathbb{R}$.

In other words, we assume that the right-hand side $f$ may be decomposed into the difference of two nondecreasing (in general discontinuous) functions. Hypothesis (H1) includes condition (1.2) as a special case by taking for $h$ the linear function $h(s)=M s$. Finally, a sufficient condition will be given which enables us to weaken the one-sided continuity assumption on $g$ such that the second example given above which involves the signum nonlinearity can also be taken into account.

2. Notations and the main result. Let $L^{2}(\Omega)$ be partially ordered by $u \leq w$ if and only if $w-u \in L_{+}^{2}(\Omega)$, where $L_{+}^{2}(\Omega)$ denotes the set of nonnegative elements of $L^{2}(\Omega)$. This relation defines a partial ordering also in the Sobolev space $H^{1}(\Omega)$ of square integrable functions having first generalized square integrable derivatives. By $H_{0}^{1}(\Omega)$ we denote the space of all the functions of $H^{1}(\Omega)$ with generalized homogeneous boundary values. The dual space of $H_{0}^{1}(\Omega)$ is denoted by $H^{-1}(\Omega)$. Let $a$ be the bilinear form associated with the elliptic differential operator $A$ by

$$
a(u, w)=\int_{\Omega} a_{i j} \frac{\partial u}{\partial x_{i}} \frac{\partial w}{\partial x_{j}} d x \quad \text { for } u, w \in H^{1}(\Omega) .
$$

Due to the assumptions on the coefficients $a_{i j}$, i.e. $a_{i j} \in L^{\infty}(\Omega)$ and $a_{i j}(x) \xi_{i} \xi_{j} \geq$ $\nu|\xi|^{2}$ for all $\xi \in \mathbb{R}^{n}$ and a.a. $x \in \Omega$, the bilinear form is bounded and coercive in $H_{0}^{1}(\Omega)$.

To motivate our definition of solution of the BVP (1.1) which will be given later let us consider two special cases of right-hand side functions $f$ satisfying hypothesis (H1).

Assume that in (H1) the function $h$ is continuous. In this case the existence of solutions lying between upper an lower solutions can be proved by monotone iteration. However, if $h$ is discontinuous then this result is no longer true and it 
may happen that there are no solutions. To illustrate this fact we consider the following BVP:

$$
-\Delta u=f(u) \quad \text { in } \Omega, \quad u=0 \quad \text { on } \partial \Omega,
$$

where $s \rightarrow f(s)$ is defined by $f(s)=1$ if $s \leq 0, f(s)=0$ if $s>0$. One readily verifies that hypothesis (H1) is satisfied by taking $h(s)=-f(s)$ and $g(s) \equiv 0$. An upper solution $\bar{u}$ and a lower solution $\underline{u}$ of the BVP (2.1) satisfying $\underline{u} \leq \bar{u}$ can be taken to be $\bar{u}=c=$ const $>0, \underline{u}=-c$. Now we show by contradiction that the BVP (2.1) has no solution.

Suppose there were a (possibly generalized) solution $u \in L^{p}(\Omega)$ for some $p>1$. Since $f(u) \in L^{\infty}(\Omega)$ one readily verifies that $u$ must be at least an element of $H_{0}^{1}(\Omega)$. Thus the following integral relation holds:

$$
\int_{\Omega} \nabla u \nabla \varphi d x=\int_{\Omega} f(u) \varphi d x \quad \text { for all } \varphi \in H_{0}^{1}(\Omega) .
$$

This implies by taking $\varphi=u$ the inequalities

$$
0 \leq \int_{\Omega}(\nabla u)^{2} d x=\int_{\Omega} f(u) u d x \leq 0
$$

and thus $u=0$, contrary to $u$ being a solution.

In order to deal with the BVP (2.1) we have to extend the notion of solution, and instead of the differential equation (2.1) we consider the associated differential inclusion of the form

$$
-\Delta u+\beta(u) \ni 0 \quad \text { in } \Omega, \quad u=0 \quad \text { on } \partial \Omega,
$$

where $\beta: \mathbb{R} \rightarrow 2^{\mathbb{R}}$ denotes the maximal monotone graph in $\mathbb{R}^{2}$ (m.m.g.) generated by the nondecreasing function $h$ which is given in our example by

$$
\beta(s)= \begin{cases}\{-1\}, & s<0 \\ {[-1,0],} & s=0, \\ \{0\}, & s>0\end{cases}
$$

One easily verifies that $u(x) \equiv 0$ is a solution of $(2.2)$ within the order interval $[-c, c]$, and moreover that this solution is unique.

Thus, in what follows, instead of the BVP (1.1) we shall consider the associated differential inclusion problem of the form

$$
A u+\beta(u) \ni g(u) \text { in } \Omega, \quad u=0 \quad \text { on } \partial \Omega,
$$

where $\beta: \mathbb{R} \rightarrow 2^{\mathbb{R}}$ denotes the m.m.g. generated by the nondecreasing function $h$. Here the m.m.g. $\beta$ is given by $\beta(s):=\left[h_{l}(s), h_{r}(s)\right]$ for all $s \in \mathbb{R}$, where $h_{l}(s)$ and $h_{r}(s)$ are the left- and right-sided limits of the function $h$ at $s$, respectively.

In general, the inclusion (2.3) does not hold pointwise, since the coefficients $a_{i j}$ are only from $L^{\infty}(\Omega)$. An appropriate weak formulation of (2.3) is given by the following definition. 
Definition 2.1. A function $u \in H_{0}^{1}(\Omega)$ is called a solution of the $B V P(2.3)$ if there exists $v \in L^{2}(\Omega)$ such that

(i) $v(x) \in \beta(u(x))$ a.e. in $\Omega$,

(ii) $a(u, w)+\int_{\Omega} v w d x=\int_{\Omega} g(u) w d x$ for all $w \in H_{0}^{1}(\Omega)$.

Next we extend the notions of upper and lower solutions known for differential equations to the case of differential inclusions.

Definition 2.2. A function $\bar{u} \in H^{1}(\Omega)$ is called an upper solution of the $B V P(2.3)$ if there exists $\bar{v} \in L^{2}(\Omega)$ such that

(i) $\bar{v}(x) \in \beta(\bar{u}(x))$ a.e. in $\Omega$,

(ii) $\bar{u} \geq 0$ on $\partial \Omega$,

(iii) $a(\bar{u}, w)+\int_{\Omega} \bar{v} w d x \geq \int_{\Omega} g(\bar{u}) w d x$ for all $w \in H_{0}^{1}(\Omega) \cap L_{+}^{2}(\Omega)$.

A lower solution $\underline{u}$ is defined similarly by reversing the inequality sign in (ii), (iii).

In the sequel we are concerned with the BVP (2.3) assuming a further hypothesis:

(H2) $\bar{u}$ and $\underline{u} \in H^{1}(\Omega)$ are an upper and a lower solution, respectively, of the original BVP (1.1) such that $\underline{u} \leq \bar{u}$ and

$$
f(\bar{u}), f(\underline{u}), h_{r}(\bar{u}), h_{l}(\underline{u}) \in L^{2}(\Omega) .
$$

The following corollary is easy to see and its proof can be omitted.

COROllary 2.3. Let hypotheses (H1) and (H2) be satisfied. Then the upper and lower solutions $\bar{u}$ and $\underline{u}$ of the BVP (1.1) are upper and lower solutions of the associated $B V P(2.3)$ in the sense of Definition 2.2. Each solution $u \in[\underline{u}, \bar{u}]$ of the BVP (1.1) is a solution of the BVP (2.3) in the sense of Definition 2.1, whereas the converse is, in general, not true as can be seen by the example given above.

The main result of this paper is

THEOREM 2.4. Let hypothesis (H1) with g being right (resp. left) continuous and hypothesis (H2) be satisfied. Then the BVP (2.3) has a greatest solution $u^{*}$ (resp. smallest solution $u_{*}$ ) within $[\underline{u}, \bar{u}]$ in the sense that if $u \in H_{0}^{1}(\Omega)$ is any other solution of $(2.3)$ in $[\underline{u}, \bar{u}]$ then $u \in\left[\underline{u}, u^{*}\right]$ (resp. $\left.\left[u_{*}, \bar{u}\right]\right)$. These extremal solutions can be constructed by monotone iteration.

The proof of Theorem 2.4 is mainly based on transforming the BVP (2.3) into an equivalent variational inequality (VI) and on the application of monotone iteration. To this end some preliminaries are needed.

3. Auxiliary results. The following property of a m.m.g. in $\mathbb{R}^{2}$ can be found e.g. in [10, Chap. 4]. 
Lemma 3.1. Let $\beta: \mathbb{R} \rightarrow 2^{\mathbb{R}}$ be any m.m.g. in $\mathbb{R}^{2}$. Then there exists a convex, proper and lower semicontinuous (l.s.c.) function $j: \mathbb{R} \rightarrow(-\infty,+\infty]$ such that $\beta$ is the subdifferential of $j$, i.e. $\beta=\partial j$. The function $j$ is uniquely determined up to an additive constant and it is superpositionally measurable.

From (1.3) we see that the function $h$ of hypothesis (H1) can always be chosen such that $h(0)=0$. This implies that the m.m.g. $\beta$ generated by $h$ has the following properties:

$$
0 \in \beta(0), \quad \beta(r) \neq \emptyset \quad \text { for all } r \in \mathbb{R} .
$$

Further we may assume

$$
j(0)=0,
$$

since the function $j$ related to $\beta$ according to Lemma 3.1 must be finite for all $r \in \mathbb{R}$ due to (3.1), and since $j$ is uniquely determined up to an additive constant. From the definition of the subgradient of $j$ at $r=0$ and from (3.1), (3.2) it follows that

$$
j(s) \geq 0 \quad \text { for all } s \in \mathbb{R} .
$$

Further, we define a functional $J: L^{2}(\Omega) \rightarrow(-\infty,+\infty]$ associated with $j$ by

$$
J(v)= \begin{cases}\int_{\Omega} j(v(x)) d x & \text { for } j(v(\cdot)) \in L^{1}(\Omega), \\ +\infty, & \text { otherwise. }\end{cases}
$$

Then $J$ is convex, proper and l.s.c. (cf. [2, p. 102]), and the restriction of $J$ to $H_{0}^{1}(\Omega)$ shares the same properties. Moreover, due to (3.2) and (3.3),

$$
J(0)=0, \quad J(v) \geq 0 \quad \text { for all } v \in L^{2}(\Omega) .
$$

Now we associate with the $\operatorname{BVP}(2.3)$ the following VI: Find $u \in H_{0}^{1}(\Omega)$ such that for all $w \in H_{0}^{1}(\Omega)$,

$$
a(u, w-u)+J(w)-J(u) \geq \int_{\Omega} g(u)(w-u) d x .
$$

The next lemma asserts that the VI (3.5) is equivalent to the BVP (2.3).

Lemma 3.2. Let the hypotheses of Theorem 2.4 be satisfied. Then $u \in[\underline{u}, \bar{u}]$ is a solution of the BVP (2.3) if and only if $u$ is a solution of the VI (3.5).

Proof. a) Let $u \in[\underline{u}, \bar{u}]$ be a solution of (3.5). By means of the subdifferential of the convex and l.s.c. functional $J: H_{0}^{1}(\Omega) \rightarrow(-\infty,+\infty]$ an equivalent formulation of (3.5) is given by

$$
-A u+g(u) \in \partial J(u) \quad \text { in } H^{-1}(\Omega) .
$$

By Brezis [3, Corollaire 1] any subgradient $v \in \partial J(u)$ at $u \in H_{0}^{1}(\Omega)$ belongs to $L^{1}(\Omega)$ and satisfies $v(x) \in \beta(u(x))$ a.e. in $\Omega$. Since $\beta(u(x)):=\left[h_{l}(u(x)), h_{r}(u(x))\right]$, the function $v$ may be bounded from above and below by $L^{2}$-functions $h_{r}(\bar{u})$ 
and $h_{l}(\underline{u})$, respectively. Thus $v \in L^{2}(\Omega)$. From (H1) and (H2) it follows that $g(u) \in L^{2}(\Omega)$. Thus from (3.6) we obtain

$$
-A u+g(u)=v \quad \text { in } H^{-1}(\Omega),
$$

or, equivalently,

$$
a(u, w)+\int_{\Omega} v w d x=\int_{\Omega} g(u) w d x
$$

for all $w \in H_{0}^{1}(\Omega)$, which means that $u$ is a solution of (2.3).

b) Conversely, let $u$ be a solution of $(2.3)$ in $[\underline{u}, \bar{u}]$. Then by definition there exists $v \in L^{2}(\Omega)$ such that $v \in \beta(u)=\partial j(u)$ and (3.7) holds. From $v(x) \in$ $\partial j(u(x))$ a.e. in $\Omega$ we obtain by definition of the subgradient that

$$
j(s) \geq j(u(x))+v(x)(s-u(x))
$$

for all $s \in \mathbb{R}$. In particular, we may take $s=w(x)$ for a.e. $x \in \Omega$ with $w \in H_{0}^{1}(\Omega)$. Integrating (3.8) over $\Omega$ with $s=w(x)$ yields

$$
J(w) \geq J(u)+\int_{\Omega} v(w-u) d x .
$$

Replacing $w$ in (3.7) by $w-u$ and taking (3.9) into account, it follows that $u$ satisfies (3.5).

COROLlary 3.3. Under the hypotheses of Theorem 2.4 the extremal solutions of the BVP (2.3) and the VI (3.5) within the order interval $[\underline{u}, \bar{u}]$ coincide, if they exist.

4. Proof of the main result. We assume the function $g$ to be right continuous and prove the existence of the greatest solution in $[\underline{u}, \bar{u}]$ of the VI (3.5) which by Corollary 3.3 is also the greatest solution of the BVP $(2.3)$. The proof will be carried out in several steps.

Step 1. Let us define an operator $T$ which assigns to each $z \in[\underline{u}, \bar{u}]$ the unique solution $u=T z$ of the following VI: Find $u \in H_{0}^{1}(\Omega)$ such that for all $w \in H_{0}^{1}(\Omega)$,

$$
a(u, w-u)+J(w)-J(u) \geq \int_{\Omega} g(z)(w-u) d x .
$$

By $(\mathrm{H} 1)$ and $(\mathrm{H} 2)$ it follows that $g(z) \in L^{2}(\Omega)$ for all $z \in[\underline{u}, \bar{u}]$. In view of the above-mentioned properties of the bilinear for $a$ and the functional $J$ the existence of a solution $u$ of (4.1) follows by standard theory of variational inequalities (cf. [14, Chap. 54]). The uniqueness of the solution is due to the strong positivity of $a$. Thus the operator $T$ is well defined.

Step 2. The following estimate holds:

$$
\|T z\|_{H^{1}(\Omega)} \leq c \quad \text { for each } z \in[\underline{u}, \bar{u}] .
$$


Taking $w=0$ as special test function in (4.1) we obtain, by means of (3.4),

$$
a(u, u) \leq \int_{\Omega} g(z) u d x \leq\|g(z)\|_{L^{2}(\Omega)}\|u\|_{H^{1}(\Omega)} .
$$

Since

$$
\|g(z)\|_{L^{2}(\Omega)} \leq\|g(\bar{u})\|_{L^{2}(\Omega)}+\|g(\underline{u})\|_{L^{2}(\Omega)}
$$

for each $z \in[\underline{u}, \bar{u}]$, we obtain the estimate (4.2) by using $a(u, u) \geq \nu\|u\|_{H^{1}(\Omega)}^{2}$.

St ep 3. We have

$$
u=T z \in[\underline{u}, \bar{u}] \quad \text { for each } z \in[\underline{u}, \bar{u}] .
$$

We show that $u \leq \bar{u}$; the proof of $\underline{u} \leq u$ is similar. By Corollary 2.3, $\bar{u}$ is an upper solution of (2.3), and thus $\bar{u}$ satisfies $\bar{u} \geq 0$ on $\partial \Omega$ and

$$
a(\bar{u}, w)+\int_{\Omega} \bar{v} w d x \geq \int_{\Omega} g(\bar{u}) w d x
$$

for all $w \in H_{0}^{1}(\Omega) \cap L_{+}^{2}(\Omega)$, where

$$
\bar{v} \in \beta(\bar{u})=\partial j(\bar{u}) .
$$

From (4.5) we get

$$
j(s) \geq j(\bar{u}(x))+\bar{v}(x)(s-\bar{u}(x))
$$

for all $s \in \mathbb{R}$ and a.e. $x \in \Omega$. Setting $w^{+}=\max \{w, 0\}$, and choosing $s=\bar{u}(x)+$ $(u(x)-\bar{u}(x))^{+}$in (4.6), we get by integration over $\Omega$,

$$
J\left(\bar{u}+(u-\bar{u})^{+}\right)-J(\bar{u}) \geq \int_{\Omega} \bar{v}(u-\bar{u})^{+} d x .
$$

Replacing $w$ in (4.4) by the special test function $(u-\bar{u})^{+}$, and taking (4.7) into account we obtain

$$
a\left(\bar{u},(u-\bar{u})^{+}\right)+J\left(\bar{u}+(u-\bar{u})^{+}\right)-J(\bar{u}) \geq \int_{\Omega} g(\bar{u})(u-\bar{u})^{+} d x .
$$

Since $u$ satisfies (4.1), for the special test function $w=u-(u-\bar{u})^{+}$we get

$$
a\left(u,-(u-\bar{u})^{+}\right)+J\left(u-(u-\bar{u})^{+}\right)-J(u) \geq-\int_{\Omega} g(z)(u-\bar{u})^{+} d x .
$$

From the monotonicity of $g$ it follows by means of (4.8) and (4.9) that

$$
a\left(\bar{u}-u,(u-\bar{u})^{+}\right)+\left[J\left(\bar{u}+(u-\bar{u})^{+}\right)-J(\bar{u})+J\left(u-(u-\bar{u})^{+}\right)-J(u)\right] \geq 0 .
$$

By elementary means one can show that the expression in brackets in (4.10) is zero. This implies in view of $a\left(w, w^{+}\right)=a\left(w^{+}, w^{+}\right)$the inequality

$$
a\left((u-\bar{u})^{+},(u-\bar{u})^{+}\right) \leq 0 .
$$

Thus $(u-\bar{u})^{+}=0$ or $u \leq \bar{u}$. 
Step 4. By a technique similar to Step 3 we can show that $T$ defines a nondecreasing mapping from $[\underline{u}, \bar{u}]$ to itself.

Step 5: Existence of a solution of the VI (3.5). By means of the operator $T$ we define an iteration of the form

$$
u^{n+1}=T u^{n} .
$$

Starting the iteration with $u^{0}=\bar{u}$ and applying Steps 3 and 4 we obtain a nonincreasing sequence $\left\{u^{n}\right\}$ of iterates which satisfy $u^{n} \in[\underline{u}, \bar{u}]$. According to Step 2 there is a constant $c$ such that

$$
\left\|u^{n}\right\|_{H^{1}(\Omega)} \leq c \quad \text { for all } n .
$$

The monotonicity and the uniform estimate (4.12) of the sequence $\left\{u^{n}\right\}$ as well as the compact imbedding $H^{1}(\Omega) \rightarrow L^{2}(\Omega)$ ensure the following convergence properties:

(i) $u^{n} \rightarrow u$ strongly in $L^{2}(\Omega)$,

(ii) $u^{n} \rightarrow u$ weakly in $H_{0}^{1}(\Omega)$.

By the definition of $T$ we have

$$
a\left(u^{n+1}, w-u^{n+1}\right)+J(w)-J\left(u^{n+1}\right) \geq \int_{\Omega} g\left(u^{n}\right)\left(w-u^{n+1}\right) d x .
$$

The functional $w \rightarrow a(w, w)$ is convex and continuous and $J$ is convex and l.s.c. This implies that each of the functionals is weakly sequentially l.s.c. (cf. [14, Chap. 37]), which enables us to pass to the limit in (4.13) to obtain

$$
a(u, w-u)+J(w)-J(u) \geq \lim _{n \rightarrow \infty} \int_{\Omega} g\left(u^{n}\right)\left(w-u^{n+1}\right) d x .
$$

Since $\left\{u^{n}\right\}$ is nonincreasing and $g$ is assumed to be right continuous, from (i) by Lebesgue's theorem we obtain

$$
\lim _{n \rightarrow \infty} \int_{\Omega} g\left(u^{n}\right)\left(w-u^{n+1}\right) d x=\int_{\Omega} g(u)(w-u) d x
$$

(cf. [4]). From (4.14) and (4.15) it follows that the limit function $u$ is a solution of $(3.5)$ within $[\underline{u}, \bar{u}]$.

S t e p 6 . In order to show that $u$ obtained by the iteration (4.11) is the greatest solution $u^{*}$ of $(3.5)$ in $[\underline{u}, \bar{u}]$, take any other solution $\widehat{u} \in[\underline{u}, \bar{u}]$ of (3.5). Then $\widehat{u}$ is in particular a lower solution of (2.3) satisfying $\widehat{u} \leq \bar{u}$. Starting again the iteration (4.11) with $u^{0}=\bar{u}$ one immediately sees that the iterates $u^{n}$ also belong to $[\widehat{u}, \bar{u}]$. Thus $u$ is contained in $[\widehat{u}, \bar{u}]$, i.e. $\widehat{u} \leq u$ and $u=u^{*}$ is the greatest solution.

Analogously, we obtain the smallest solution $u_{*}$ of $(2.3)$ in $[\underline{u}, \bar{u}]$ if we start the iteration (4.11) with the lower solution $\underline{u}$ provided that $g$ is left continuous. This completes the proof. 
Rem ark 4.1. With slight modifications general uniformly elliptic operators of the form

$$
A u=-\frac{\partial}{\partial x_{i}}\left(a_{i j} \frac{\partial u}{\partial x_{j}}\right)+a_{i} \frac{\partial u}{\partial x_{i}}+a_{0} u
$$

can as well be taken into account. In the same way one can deal with quasilinear elliptic operators $A$ in divergence form provided they generate strongly monotone and coercive operators acting from $H_{0}^{1}(\Omega)$ into its dual.

Rem ark 4.2. The method developed in this paper is constructive and may be used for computational purposes. At least in the one-dimensional case numerical procedures based on monotone discretization techniques can be established. In case that the right-hand side of (1.1) is continuous and satisfies a one-sided Lipschitz condition, monotone discretization for generating enclosures have been developed by C. Großmann e.g. in [12] (see also the paper by Großmann in this volume).

Remark 4.3. The existence of extremal solutions within $[\underline{u}, \bar{u}]$ holds even for the continuously perturbed BVP

$$
A u+p(u)+\beta(u) \ni g(u) \quad \text { in } \Omega, \quad u=0 \quad \text { on } \partial \Omega,
$$

where $A, \beta$ and $g$ are as before and $p: \mathbb{R} \rightarrow \mathbb{R}$ is an arbitrary but continuous function which is only supposed to be $L^{2}$-bounded with respect to $[\underline{u}, \bar{u}]$. However, the proof of this assertion is no longer constructive, since now the operator $T$ defined above becomes multivalued. The results in [6] are closely related to the BVP (4.16).

Rem ark 4.4. The assumption of right (resp. left) continuity imposed on the nonlinearity $g$ has been made for technical reasons in order to pass to the limit on the right-hand side of (4.13). If $g$ is not one-sided continuous then by the monotonicity of $g$ at least its one-sided limits, i.e. $g_{l}$ and $g_{r}$, exist. Thus we may apply Theorem 2.4 to the following auxiliary BVP associated with the BVP (2.3):

$$
A u+\beta(u) \ni g_{r}(u)\left(\text { resp. } g_{l}(u)\right) \quad \text { in } \Omega, \quad u=0 \quad \text { on } \partial \Omega .
$$

Let $\Omega_{s}$ be the level set of $u$ defined by $\Omega_{s}=\{x \in \Omega \mid u(x)=s\}$. One can show that each solution of (4.17) is a solution of (2.3) provided that the level sets $\Omega_{s}$ of the solutions of (4.17) have $L$-measure zero at the points $s$ where $g_{r}(s) \neq g(s)$ (resp. $\left.g_{l}(s) \neq g(s)\right)$. In the case that the coefficients $a_{i j}$ are sufficiently smooth $m\left(\Omega_{s}\right)=0$ holds provided that $g_{r}(s) \notin \beta(s)$ (resp. $\left.g_{l}(s) \notin \beta(s)\right)$.

\section{References}

[1] R. Aris, The Mathematical Theory of Diffusion and Reaction in Permeable Catalysts, Vols. 1 and 2, Clarendon Press, Oxford 1975. 
[2] V. Barbu and Th. Precupanu, Convexity and Optimization in Banach Spaces, Editura Academiei, Bucureşti, and Sijthoff, Noordhoff 1978.

[3] H. Brezis, Intégrales convexes dans les espaces de Sobolev, Israel J. Math. 13 (1972), 9-23.

[4] S. Carl, Ein konstruktiver Existenzsatz für Randwertprobleme elliptischer Differentialgleichungen zweiter Ordnung mit unstetiger Nichtlinearität, Math. Nachr. 138 (1988), 5565 .

[5] S. Carl and S. Heikkilä, An existence result for elliptic differential inclusions with discontinuous nonlinearity, Nonlinear Anal. 18 (1992), 471-479.

[6] - - - On extremal solutions of an elliptic boundary value problem involving discontinuous nonlinearities, Differential Integral Equations 5 (1992), 581-589.

[7] P. W. Davis and B. A. Fleishman, A discontinuous nonlinear problem: stability and convergence of iterations in a finite number of steps, Appl. Anal. 23 (1986), 139-157.

[8] J. Deuel and P. Hess, A criterion for the existence of solutions of nonlinear elliptic boundary value problems, Proc. Roy. Soc. Edinburgh 74A (1974/75), 49-54.

[9] J. I. Diaz and J. Hernandez, On the existence of a free boundary for a class of reactiondiffusion systems, SIAM J. Math. Anal. 15 (1984), 670-685.

[10] J. I. Diaz, Nonlinear Partial Differential Equations and Free Boundaries, Vol. 1: Elliptic Equations, Res. Notes in Math. 106, Pitman, Boston 1985.

[11] B. A. Fleishman, Convex superposition in piecewise-linear systems, J. Math. Anal. Appl. 6 (1963), 182-189.

[12] C. Großmann, Monotone discretization of two-point boundary value problems and related numerical methods, in: Discretization in Differential Equations and Enclosures, E. Adams, R. Ansorge, C. Großmann and H.-G. Roos (eds.), Akademie-Verlag, Berlin 1987, 99-122.

[13] S. Heik kilä, On an elliptic boundary value problem with discontinuous nonlinearity, Appl. Anal. 37 (1990), 183-189.

[14] E. Zeidler, Nonlinear Functional Analysis and its Applications III: Variational Methods, Springer, New York 1985. 